\title{
Utilizing slide-ring cross-linkers in polysiloxane networks for softer dielectric elastomer actuators
}

Tran, Jakob-Anhtu; Madsen, Jeppe ; Skov, Anne Ladegaard

Published in:

Electroactive Polymer Actuators and Devices (EAPAD) XXII

Link to article, DOI:

$10.1117 / 12.2557355$

Publication date:

2020

Document Version

Publisher's PDF, also known as Version of record

Link back to DTU Orbit

Citation (APA):

Tran, J-A., Madsen, J., \& Skov, A. L. (2020). Utilizing slide-ring cross-linkers in polysiloxane networks for softer dielectric elastomer actuators. In Y. Bar-Cohen, I. A. Anderson, \& H. R. Shea (Eds.), Electroactive Polymer Actuators and Devices (EAPAD) XXII [1137518] SPIE - International Society for Optical Engineering. Proceedings of SPIE - The International Society for Optical Engineering Vol. 11375 https://doi.org/10.1117/12.2557355

\section{General rights}

Copyright and moral rights for the publications made accessible in the public portal are retained by the authors and/or other copyright owners and it is a condition of accessing publications that users recognise and abide by the legal requirements associated with these rights.

- Users may download and print one copy of any publication from the public portal for the purpose of private study or research.

- You may not further distribute the material or use it for any profit-making activity or commercial gain

- You may freely distribute the URL identifying the publication in the public portal 


\section{Utilizing slide-ring cross-linkers in polysiloxane networks for softer dielectric elastomer actuators}

Tran, Jakob-Anhtu, Madsen, Jeppe, Skov, Anne Ladegaard

Jakob-Anhtu Tran, Jeppe Madsen, Anne Ladegaard Skov, "Utilizing slide-ring cross-linkers in polysiloxane networks for softer dielectric elastomer actuators," Proc. SPIE 11375, Electroactive Polymer Actuators and Devices (EAPAD) XXII, 1137518 (22 April 2020); doi: 10.1117/12.2557355

Event: SPIE Smart Structures + Nondestructive Evaluation, 2020, Online Only, California, United States 


\title{
Utilizing slide-ring cross-linkers in polysiloxane networks for softer dielectric elastomer actuators
}

\author{
Jakob-Anhtu Tran ${ }^{1}$, Jeppe Madsen ${ }^{1}$, Anne Ladegaard Skov ${ }^{1}$ \\ ${ }^{1}$ Danish Polymer Center, Department of Chemical and Biochemical Engineering, Technical \\ University of Denmark, Søltofts Plads, Building 227, 2800 Kgs. Lyngby, Denmark
}

\begin{abstract}
Slide-ring elastomers have mobile cross-links that can slide on their axial polymers in a manner similar to a pulley on a zip line. This supramolecular network structure imparts unique mechanical properties to the elastomers, such as high deformability and low hysteresis upon cyclic loading, that are often favorable for dielectric elastomer actuators (DEAs). The utilization of this type of dynamic network for actuation has been limited by the low compatibility of slide-ring materials and common elastomer platforms used in DEAs. Here, a synthetic pathway is proposed to allow for incorporation of slide-ring cross-linkers into silicone networks.
\end{abstract}

Keywords: Silicone, slide-ring elastomer, DEA, polyrotaxane

\section{INTRODUCTION}

Dielectric elastomer actuators (DEAs) have garnered considerable interest due to the possibilities they present as lightweight, flexible energy transducers with fast response rates. The applications for this type of innovative actuator range from soft robotics and artificial muscles to self-cleaning surfaces. ${ }^{1}$ Silicone elastomers are commonly used for DEA fabrication because of their advantageous properties for actuation, such as a broad operating temperature, low mechanical loss, and biocompatibility. ${ }^{2}$ When tailoring silicones for improved actuation at low voltages, two specific properties are sought after: high dielectric permittivity and low Young's modulus. However, formulating softer elastomers from traditional curing schemes usually presents a tradeoff with lower mechanical integrity and higher viscous loss. ${ }^{3}$

As an alternative to common elastomers, slide-ring materials demonstrate a novel type of network structure in which, instead of traditional fixed cross-links, the junctures can slide on a molecular level, enabling a unique mechanical behavior often described as the pulley effect. ${ }^{4}$ When slide-ring materials are deformed, the sliding cross-links are able to dissipate the internal stress in a similar fashion to a pulley sliding on a rope. This molecular scale phenomenon has been credited for the softness and high maximum elongation of slide-ring elastomers. ${ }^{5}$ Additionally, slide-ring elastomers have been proven to exhibit low mechanical hysteresis, which has been attributed to the sliding cross-links' ability to easily shift back to their relaxed state in a manner similar to a one-dimensional air spring. ${ }^{6}$

Polyethylene glycol (PEG) and $\alpha$-cyclodextrin $(\alpha-\mathrm{CD})$ based polyrotaxanes have shown promise as a versatile platform for slide-ring materials due to their commercially available components and relative ease of production. The supramolecular necklace structures form spontaneously when the PEG axis polymers and $\alpha$-CD rings are dissolved in water. It has been found that the threading process is largely driven by hydrogen-bond formation between rings aligned by the axis polymer. ${ }^{7,8}$ The threaded complexes are then further stabilized by the formation of ordered hexagonal packing structures with neighboring molecules, which causes the aggregates to precipitate. ${ }^{9,10}$ Subsequent capping of the axis polymers with bulky stopper groups is then necessary to avoid dissociation of the rings and to form the final polyrotaxanes.

In order to create slide-ring materials, it is crucial that the polyrotaxane rings can slide freely on their axis polymers, which is not possible if they are densely clustered in crystalline domains. Disruption of the hydrogen bonding between the rings is thus necessary to enable their free axial movement. This disruption is generally achieved by substituting the hydroxyl groups on $\alpha$-CD rings. ${ }^{11}$ It is also possible to increase the solubility of the modified polyrotaxanes in common 
organic solvents by altering the substitution chemistry. ${ }^{12}$ The modified rings can then be linked together to form a sliding network.

While the production of PEG/ $\alpha$-CD polyrotaxanes has reached a commercial scale, their inclusion in silicone networks has been limited. ${ }^{13,14}$ Here, we have proposed a synthetic pathway to produce polyrotaxane cross-linkers that contain vinyl groups, allowing them to be incorporated into polysiloxane networks through hydrosilylation. This type of curing chemistry is commonly used in silicone elastomers due to its high efficiency and selectivity. ${ }^{15}$

\section{EXPERIMENTAL}

\subsection{Materials}

Polyethylene glycol (PEG 35000) was purchased from Fluka. 1H-Benzotriazol-1-yloxytripyrrolidinophosphonium Hexafluorophosphate (PyBOP) was purchased from Tokyo Chemical Industry Co., Ltd. 1,3-divinyl-1,1,3,3tetramethyldisilazane, platinum-divinyltetramethylsiloxane complex $(3.0 \% \mathrm{Pt})$ as well as hydride and vinyl terminated polydimethylsiloxanes (PDMS $\mathrm{M}_{\mathrm{n}}=20000$ to $24000 \mathrm{~g} \mathrm{~mol}^{-1}$ ) were procured from Gelest Inc. All other chemicals were purchased from Merck KGaA and used as is unless otherwise stated.

\subsection{Characterization}

${ }^{1} \mathrm{H}-\mathrm{NMR}$ spectra were acquired on a Bruker $300 \mathrm{MHz}$ spectrometer in either deuterated DMSO or chloroform at ambient temperature unless stated otherwise, with the residual solvent used as the internal standard. Size Exclusion Chromatography (SEC) measurements were conducted with Shimadzu instrumentation (SIL-10AD autoinjector, LC10AD liquid chromatograph, RID-10A refractive index detector) with a $10 \mathrm{mM}$ LiBr DMSO eluent flow of 1.000 $\mathrm{mL} / \mathrm{min}$ at room temperature through a Millipore/Waters Ultrastyragel $10^{5} \AA ̊$ column.

Thermogravimetric analysis (TGA) was performed in air with a heating rate of $10{ }^{\circ} \mathrm{C} \mathrm{min}^{-1}$ from RT to $900{ }^{\circ} \mathrm{C}$ on Discovery TGA instrumentation from TA Instruments. The onset of thermal degradation $\left(\mathrm{T}_{\mathrm{d} 5 \%}\right)$ was determined as the temperature at $5 \%$ weight loss.

Electrical breakdown tests were performed on an in-house built device based on international standards (IEC 60,243-1 (1998) and IEC 60,243-2 (2001)). A stepwise increase of voltage was applied to the samples (50 V step s s $^{-1}$ at a rate of 0.5 steps s$^{-1}$. Film thickness was determined through optical microscopy of the film cross-section.

The tensile properties of the elastomers were measured using the Instron 3340 materials testing system (INSTRON, US). Samples of $30 \mathrm{~mm}$ length and $3.18 \mathrm{~mm}$ width were placed between two clamps with an initial separation distance of 10 $\mathrm{mm}$. The test specimens were elongated uniaxially at $50 \mathrm{~mm} \mathrm{~min}^{-1}$.

Dielectric relaxation spectroscopy (DRS) was performed at room temperature and low electrical field $\left(\sim 1 \mathrm{~V} \mathrm{~mm}^{-1}\right)$ on a Novocontrol Alpha-A high-performance frequency analyser (Concept 40 Top Class System, Novocontrol Technologies $\mathrm{GmbH} \& \mathrm{Co}$, Germany) operating in the frequency range $10^{-1}-10^{6} \mathrm{~Hz}$. The samples were approximately $0.1 \mathrm{~mm}$ thick and $20 \mathrm{~mm}$ in diameter.

\subsection{Preparation of polyrotaxanes}

To prepare the carboxylic acid terminated PEG-COOH, ten grams of PEG was oxidized in $100 \mathrm{~mL}$ of DI water with 2,2,6,6-tetramethyl-1-piperidinyloxy radical (TEMPO, $100 \mathrm{mg}, 0.64 \mathrm{mmol}), \mathrm{KBr}(116 \mathrm{mg}, 0.97 \mathrm{mmol})$ and $\mathrm{NaClO}$ (20 $\mathrm{mL}, 5 \%$ available chlorine) at a $\mathrm{pH}$ of $11-12$ for $15 \mathrm{~min}$. The oxidation was quenched with $10 \mathrm{~mL}$ of ethanol. The reaction mixture was then acidified with $\mathrm{HCl}$ to a $\mathrm{pH}$ below 2 . The product was extracted with two $100 \mathrm{~mL}$ aliquots of DCM that were then combined and evaporated in vacuo. The product was dissolved in hot ethanol and precipitated at -20 ${ }^{\circ} \mathrm{C}$. The precipitate was then washed with ethanol and dried in vacuo overnight. The end-group conversion was confirmed with ${ }^{1} \mathrm{H}-\mathrm{NMR}$ to be $>98 \%$.

3 grams of PEG-COOH and 13.7 grams of $\alpha$-cyclodextrin $(\alpha-\mathrm{CD})$ were dissolved in $100 \mathrm{~mL}$ of DI water and stirred for 15 minutes before overnight complexation at $-5{ }^{\circ} \mathrm{C}$. The complexed pseudo-polyrotaxane (PPR) was then freeze-dried and crushed into a fine powder. The PPR was then end-capped with the addition of 1-adamantylamine (169 mg, 1.1 
mmol), PyBOP (581 mg, $1.1 \mathrm{mmol})$ and $\mathrm{N}, \mathrm{N}$-diisopropylethylamine $(0.2 \mathrm{~mL}, 1.2 \mathrm{mmol})$. The mixture was dissolved in $100 \mathrm{~mL}$ of dry N, N-Dimethylformamide (DMF) and stirred overnight at RT in an inert atmosphere. The reaction mixture was then washed through centrifugation, twice with a 1 to 1 mixture of DMF/methanol and twice with methanol. The obtained solid was then dissolved in dimethylsulfoxide and precipitated in water, followed by repeated washing with water and freeze-drying.

\subsection{Preparation of vinyl functional polyrotaxanes (ViPR)}

The polyrotaxane material $(1 \mathrm{~g})$ was dissolved in dry DMSO $(40 \mathrm{~mL})$. A mixture $(4.4 \mathrm{~g}, 20.4 \mathrm{mmol})$ of 1,3-divinyl1,1,3,3-tetramethyldisilazane (DVTMDS) and 1,1,1,3,3,3-hexamethylsilazane (HMDS) was slowly added to the reaction vessel in a drop-wise manner. The ratio of the silylating agents was varied between experiments. The reaction mixture was stirred for 6 hours at $60{ }^{\circ} \mathrm{C}$, followed by an addition of dry THF $(40 \mathrm{~mL})$. The mixture then left to react for an additional 20 hours. The product was then precipitated in methanol $(800 \mathrm{~mL})$, washed with methanol and dried under vacuum, resulting in a white solid.

\subsection{Preparation of slide-ring silicone films}

Hydride terminated PDMS $\left(0.14 \mathrm{~g}, M_{n}=24000 \mathrm{~g} \mathrm{~mol}^{-1}\right)$, vinyl terminated PDMS extenders $\left(0.06 \mathrm{~g}, M_{n}=20000 \mathrm{~g}\right.$ $\left.\mathrm{mol}^{-1}\right)$ and ViPR cross-linkers $\left(0.04 \mathrm{~g}, f_{\text {vinyl }}=0.49 \mathrm{mmol} \mathrm{g}^{-1}\right)$ were dissolved in Toluene. Platinum catalyst was added (50 ppm) and the mixture was left to pre-cure for 15 minutes at $80^{\circ} \mathrm{C}$. The mixture was then cast into a Teflon mold and left to evaporate for two days before the final overnight curing at $80^{\circ} \mathrm{C}$ in an oven.

\section{RESULTS AND DISCUSSION}

\subsection{Preparation of slide-ring cross-linkers}

Polyrotaxanes consisting of polyethylene glycol (PEG) and $\alpha$-cyclodextrin ( $\alpha$-CD) were synthesized according to methods previously described in the literature ${ }^{16}$, with minor modifications. End-capping and purification of the complexed PEG and $\alpha$-CD were confirmed by size exclusion chromatography. ${ }^{1} \mathrm{H}-\mathrm{NMR}$ was then used to determine the average number of $\alpha-\mathrm{CD}$ rings threaded for each PEG chain (Figure 1). Approximately 100 rings were threaded for each polyrotaxane molecule, which corresponds to an inclusion ratio of $25 \%$. 


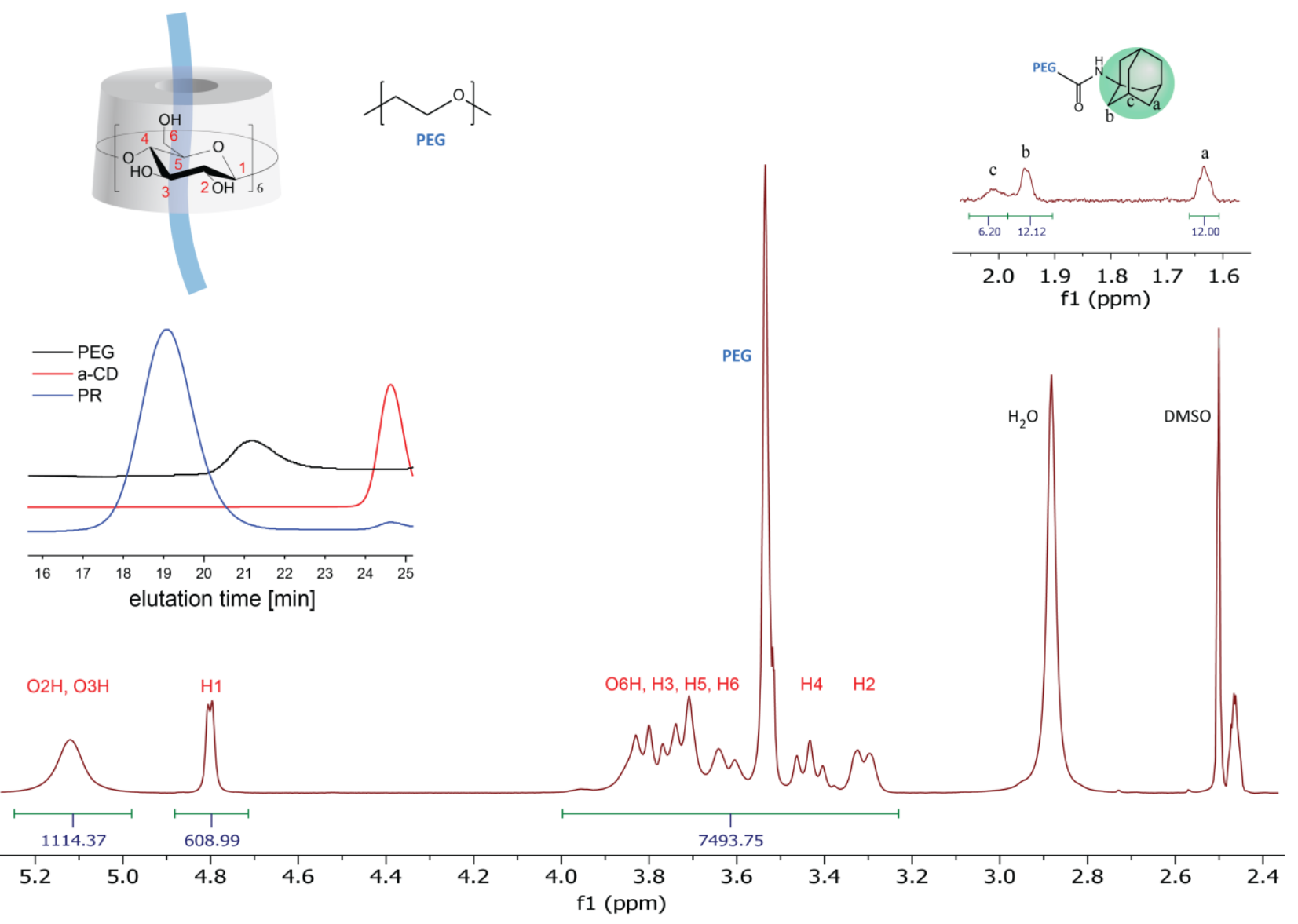

Figure 1: ${ }^{1} \mathrm{H}-\mathrm{NMR}$ spectrum (400 MHz, d6-DMSO, $80{ }^{\circ} \mathrm{C}$ ) and $\mathrm{SEC}$ analysis (detector: RI, eluent: $10 \mathrm{mM} \mathrm{LiBr}$ in DMSO) of polyrotaxanes.

The polyrotaxanes were then modified to disrupt the dense packing structure of the rings and to improve their solubility in organic solvents while also introducing reactive handles for network formation. The hydroxyl groups on the $\alpha-C D$ rings were silylated with different feed ratios of HMDS and DVTMDS to control the number of reactive vinyl groups introduced per ring (Figure 2). An average of one networking group per ring is crucial to ensure that the cross-links can slide in relation to each other instead of forming fixed junctions on a single ring. 
A)
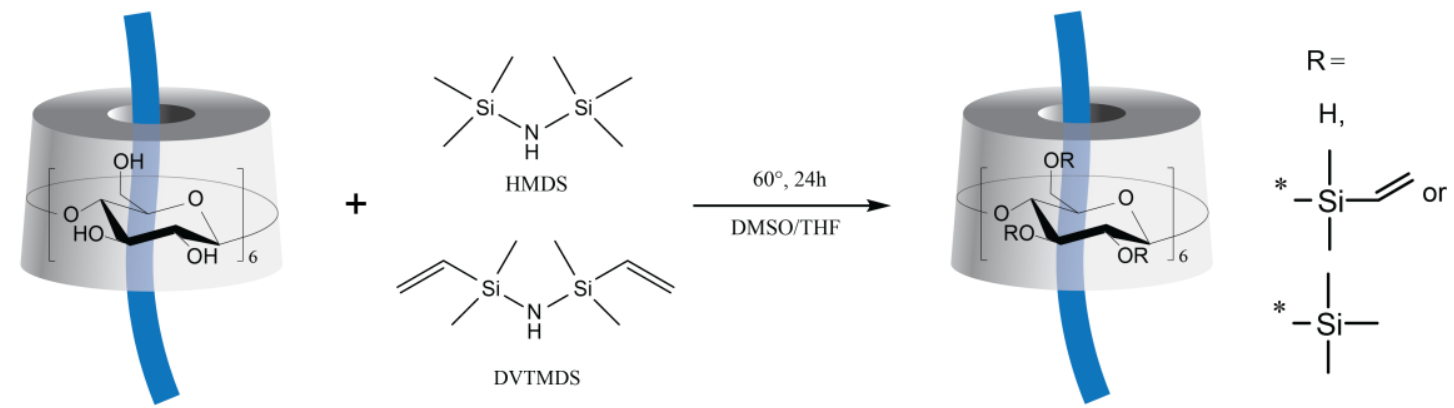

B)

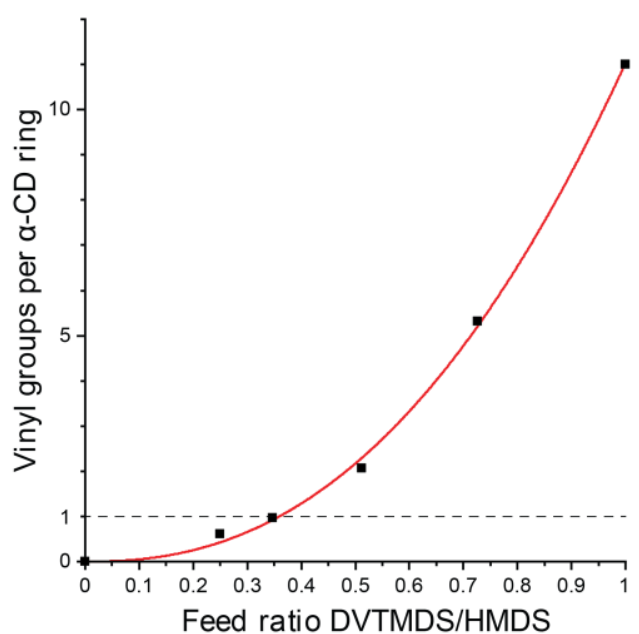

Figure 2: A) Synthesis of modified polyrotaxanes with vinyl functional rings (ViPR). B) Vinyl groups per $\alpha$-CD ring based on the feed ratio.

Altering the stoichiometric balance of the two silylating agents in the reaction feed was found to effectively control the number of vinyl functional groups per ring and ensure that the degree of substitution of the hydroxyl groups was sufficient to increase the solubility of the modified polyrotaxane (Table 1). The final polyrotaxane cross-linkers were soluble in chloroform, toluene, and tetrahydrofuran.

Table 1: Effect of the feed ratio of the silylating agents on the final functional group composition of the modified polyrotaxanes (ViPR), where TMS is the unreactive trimethylsilyl group, OH the hydroxyl, and DS the degree of substitution of each glucose unit in the ring.

Groups per $\alpha-C D$ ring

\begin{tabular}{c|ccc|c}
\hline Feed & Vinyl & TMS & OH & DS \\
\hline 0 & 0 & 12.9 & 5.1 & 2.15 \\
0.25 & 0.6 & 12.0 & 5.3 & 2.11 \\
0.35 & 1.0 & 10.5 & 6.6 & 1.91 \\
0.51 & 2.1 & 11.2 & 4.7 & 2.21 \\
0.73 & 5.3 & 8.1 & 4.6 & 2.24 \\
1 & 11.0 & 0 & 7.0 & 1.83
\end{tabular}




\subsection{Preparing slide-ring silicone elastomers}

The modified slide-ring cross-linkers were pre-cured in toluene with hydride terminated PDMS and chain extenders before solvent casting and final curing (Figure 3). The extent of the pre-curing reaction was found to be essential for ensuring a homogeneous reaction mixture in the final curing of the elastomer films. Insufficient pre-curing caused the formation of cross-linker rich macroscopic phases, while excessive pre-curing resulted in issues during the casting process due to excessive gelling.

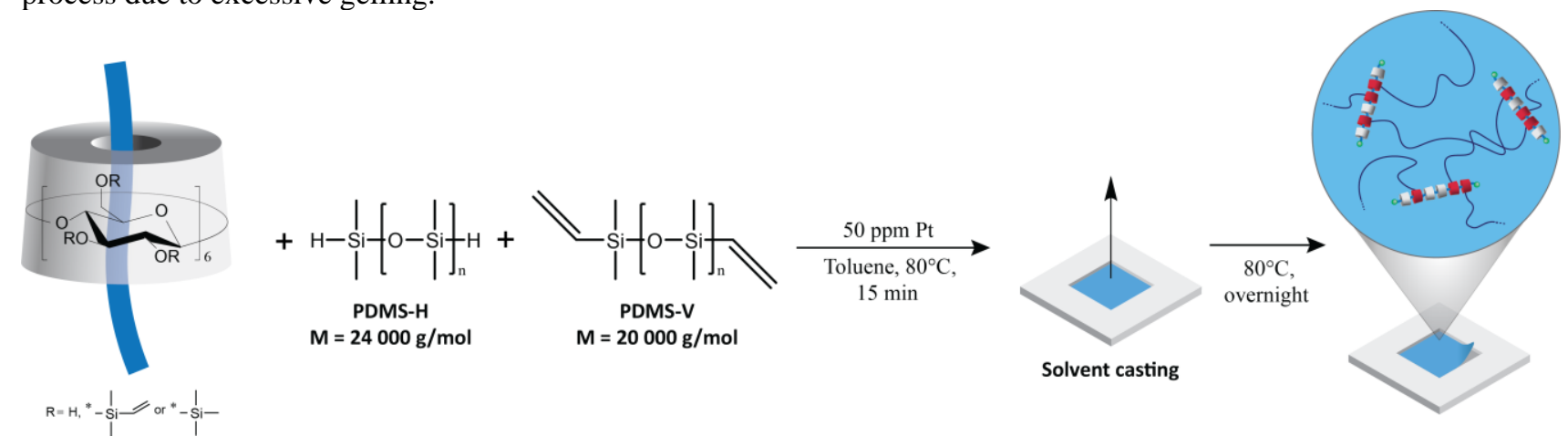

Figure 3: Synthesis of slide-ring silicone elastomers.

The cured elastomers were transparent, and no visible phase separation was observed under optical microscopy. Given the large molecular weight of the slide-ring cross-linkers $\left(M_{n}=230000 \mathrm{~g} \mathrm{~mol}^{-1}\right)$, longer linear segments were necessary to ensure that the silicone component of the system would dominate the bulk dielectric properties. Chain extenders were utilized to increase the length of the PDMS segments to approximately $64000 \mathrm{~g} \mathrm{~mol}^{-1}$, resulting in the final composition of the network being $83 \mathrm{wt} \%$ silicone.

The mechanical and dielectric properties of the slide-ring elastomer are shown in Table 2 . The dielectric permittivity and electrical breakdown strength of the films were in the same value range as pure silicone elastomers, confirming the negligible effect of the cross-linkers on the bulk properties. The Young's modulus and maximum strain of the initial slide-ring elastomers presented no improvement compared to commercial silicones. This could be caused by the formulation of the curing mixture resulting in a denser network. Alternatively, the high number of threaded rings on each axis polymer could also restrict the degree of molecular sliding possible for the rings. Further study of different curing compositions and fixed cross-linked analogues is thus necessary to confirm the intrinsic properties caused by the ring sliding.

Table 2: Mechanical and electrical properties of the slide-ring elastomers.

\begin{tabular}{cccc}
\hline $\mathrm{Y}(\mathrm{MPa})$ & $\varepsilon^{\prime}$ & $\mathrm{E}_{\mathrm{b}}(\mathrm{V} / \mu \mathrm{m})$ & Max. strain \\
\hline 1.7 & 2.6 & 37.8 & $250 \%$
\end{tabular}

\subsection{Thermal properties of the slide-ring materials}

Thermal degradation studies on the polyrotaxane materials and precursors revealed that modifying the hydroxyl groups increased the thermal stability of the polyrotaxanes (Figure 4). Substituting the hydroxyls with silyl groups increased the onset of thermal degradation by $13 \%$, while grafting polysiloxane side-chains showed an increase of $64 \%$ compared to the unmodified polyrotaxanes. However, the cured slide-ring silicone film had a slightly lower thermal stability when compared to the grafted rotaxanes. 


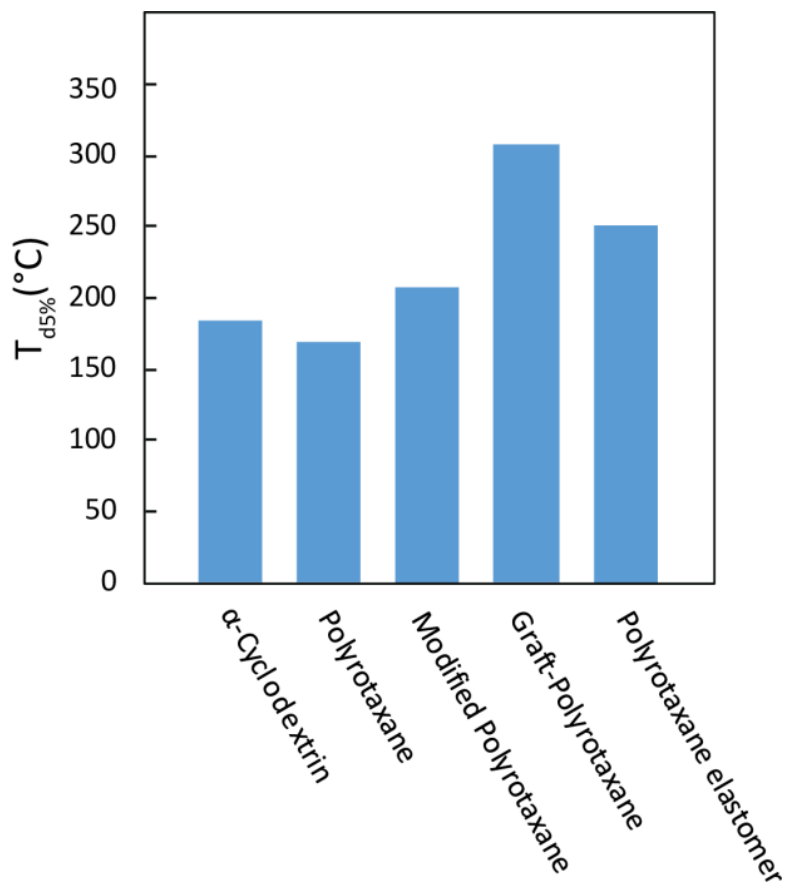

Figure 4: Thermal degradation of $\alpha$-cyclodextrin and polyrotaxane materials in inert atmosphere.

\section{CONCLUSION}

Homogeneous silicone slide-ring elastomers were successfully synthesized by modifying PEG and $\alpha$-CD based polyrotaxanes to facilitate curing with linear polysiloxanes. The modification method allowed control of cross-linking functionalities per threaded CD rings and was found to increase the thermal stability of the polyrotaxanes. The dielectric properties of the slide-ring elastomers were shown to be dictated by the silicone component of the material. A wider study of the chemistry of the linear part of the network and the inclusion of reinforcing fillers would be required to fully explore the mechanical effect of ring sliding in these novel elastomers as well as their further use as dielectric elastomer actuators. 


\section{REFERENCES}

[1] Bauer, S., Bauer-Gogonea, S., Graz, I., Kaltenbrunner, M., Keplinger, C. and Schwödiauer, R., "25th Anniversary Article: A Soft Future: From Robots and Sensor Skin to Energy Harvesters,” Adv. Mater. 26(1), 149-162 (2014).

[2] Madsen, F. B., Daugaard, A. E., Hvilsted, S. and Skov, A. L., "The Current State of Silicone-Based Dielectric Elastomer Transducers," Macromol. Rapid Commun. 37(5), 378-413 (2016).

[3] Skov, A. L. and Yu, L., "Optimization Techniques for Improving the Performance of Silicone-Based Dielectric Elastomers," Adv. Eng. Mater. 20(5), 1700762 (2018).

[4] Okumura, Y. and Ito, K., "The Polyrotaxane Gel: A Topological Gel by Figure-of-Eight Cross-links," Adv. Mater. 13(7), 485-487 (2001).

[5] Minato, K., Mayumi, K., Maeda, R., Kato, K., Yokoyama, H. and Ito, K., "Mechanical properties of supramolecular elastomers prepared from polymer-grafted polyrotaxane," Polymer (Guildf). 128, 386-391 (2017).

[6] Kato, K., Okabe, Y., Okazumi, Y. and Ito, K., "A significant impact of host-guest stoichiometry on the extensibility of polyrotaxane gels," Chem. Commun. 51(90), 16180-16183 (2015).

[7] Harada, A., Li, J. and Kamachi, M., "The molecular necklace: A rotaxane containing many threaded $\alpha$ cyclodextrins," Nature 356(6367), 325-327 (1992).

[8] Biedermann, F., Nau, W. M. and Schneider, H. J., "The Hydrophobic Effect Revisited - Studies with Supramolecular Complexes Imply High-Energy Water as a Noncovalent Driving Force," Angew. Chemie - Int. Ed. 53(42), 11158-11171 (2014).

[9] Ceccato, M., Lo Nostro, P. and Baglioni, P., " $\alpha$-cyclodextrin/polyethylene glycol polyrotaxane: A study of the threading process," Langmuir 13(9), 2436-2438 (1997).

[10] Inomata, A., Sakai, Y., Zhao, C., Ruslim, C., Shinohara, Y., Yokoyama, H., Amemiya, Y. and Ito, K., "Crystallinity and cooperative motions of cyclic molecules in partially threaded solid-state polyrotaxanes," Macromolecules 43(10), 4660-4666 (2010).

[11] Mayumi, K., Ito, K. and Kato, K., [Polyrotaxane and Slide-Ring Materials], The Royal Society of Chemistry, Cambridge, 137-139 (2016).

[12] Araki, J. and Ito, K., "Polyrotaxane derivatives. I. Preparation of modified polyrotaxanes with nonionic functional groups and their solubility in organic solvents," J. Polym. Sci. Part A Polym. Chem. 44(21), 63126323 (2006).

[13]Zhuo, Y., Li, T., Wang, F., Håkonsen, V., Xiao, S., He, J. and Zhang, Z., "An ultra-durable icephobic coating by a molecular pulley," Soft Matter 15(17), 3607-3611 (2019).

[14] Du, R., Xu, Z., Zhu, C., Jiang, Y., Yan, H. and Wu, H., "A Highly Stretchable and Self-healing Supramolecular A Highly Stretchable and Self-healing Supramolecular Elastomer based on Sliding Crosslinks and Hydrogen Bonds," Adv. Funct. Mater. 30(7), 1907139 (2020).

[15] Marciniec, B., "Functionalisation and Cross-Linking of Organosilicon Polymers," [Hydrosilylation: A Comprehensive Review on Recent Advances], B. Marciniec, Ed., Springer Netherlands, Dordrecht, 159-189 (2009).

[16] Araki, J., Zhao, C. and Ito, K., "Efficient Production of Polyrotaxanes from Cyclodextrin and Poly (ethylene glycol )," Macromolecules 38(17), 7524-7527 (2005). 